\title{
Specific markers and properties of synovial mesenchymal stem cells in the surface, stromal, and perivascular regions
}

\author{
Mitsuru Mizuno1, Hisako Katano1, Yo Mabuchi', Yusuke Ogata², Shizuko Ichinose ${ }^{1}$, Shizuka Fujii ${ }^{1}$, Koji Otabe ${ }^{1}$, \\ Keiichiro Komori ${ }^{1}$, Nobutake Ozeki ${ }^{1}$, Hideyuki Koga ${ }^{3}$, Kunikazu Tsuji ${ }^{4}$, Chihiro Akazawa ${ }^{2}$, Takeshi Muneta ${ }^{5}$ \\ and Ichiro Sekiya ${ }^{1 *}$ (D)
}

\begin{abstract}
Background: Synovial mesenchymal stem cells (MSCs) are an attractive cell source for cartilage and meniscus regeneration. Synovial tissue can be histologically classified into three regions; surface, stromal and perivascular region, but the localization of synovial MSCs has not been fully investigated. We identified markers specific for each region, and compared properties of MSCs derived from each region in the synovium.

Methods: The intensity of immunostaining with 19 antibodies was examined for surface, stromal, and perivascular regions of human synovium from six osteoarthritis patients. Specific markers were identified and synovial cells derived from each region were sorted. Proliferation, surface marker expression, chondrogenesis, calcification and adipogenesis potentials were compared in synovial MSCs derived from the three regions.

Results: We selected $\mathrm{CD} 55^{+} \mathrm{CD} 271^{-}$for synovial cells in the surface region, $\mathrm{CD} 55^{-} \mathrm{CD} 271^{-}$in the stromal region, and $\mathrm{CD}_{55^{-}} \mathrm{CD} 271^{+}$in the perivascular region. The ratio of the sorted cells to non-hematopoietic lineage cells was $5 \%$ in the surface region, $70 \%$ in the stromal region and $15 \%$ in the perivascular region. Synovial cells in the perivascular fraction had the greatest proliferation potential. After expansion, surface marker expression profiles and adipogenesis potentials were similar but chondrogenic and calcification potentials were higher in synovial MSCS derived from the perivascular region than in those derived from the surface and stromal regions.
\end{abstract}

Conclusions: We identified specific markers to isolate synovial cells from the surface, stromal, and perivascular regions of the synovium. Synovial MSCs in the perivascular region had the highest proliferative and chondrogenic potentials among the three regions.

\section{Background}

Mesenchymal stem cells (MSCs) are an attractive cell source for cell therapies. These cells participate in tissue homoeostasis, remodeling, and repair by ensuring replacement of mature cells that are lost during the course of physiological turnover, senescence, injury, or disease [1]. Along with preclinical studies, a large number of clinical trials have been conducted for cardiovascular diseases, osteoarthritis, liver disorders, graft versus host disease (GvHD), respiratory disorders, spinal cord injury,

\footnotetext{
* Correspondence: sekiya.arm@tmd.ac.jp

${ }^{1}$ Center for Stem Cell and Regenerative Medicine, Tokyo Medical and Dental

University, 1-5-45 Yushima, Bunkyo-ku, Tokyo 113-8510, Japan

Full list of author information is available at the end of the article
}

and others [2]. MSCs are found not only in bone marrow but multiple adult tissues [3-5].

MSCs are defined as non-hematopoietic-lineage, plastic-adherent, self-renewing cells that can differentiate into chondrocytes, adipocytes and osteoblasts in vitro [6, 7]. Traditionally, the isolation of MSCs has relied on their adherence to plastic dishes and colony-forming ability in an unfractionated cell population. This technique may give rise to heterogeneous cell populations in MSCs. To better characterize this heterogeneity, surface markers have been investigated for bone marrow MSCs from the osteoblast region [8], endosteum region [9], and perivascular region [10].

(c) The Author(s). 2018 Open Access This article is distributed under the terms of the Creative Commons Attribution 4.0 International License (http://creativecommons.org/licenses/by/4.0/), which permits unrestricted use, distribution, and reproduction in any medium, provided you give appropriate credit to the original author(s) and the source, provide a link to the Creative Commons license, and indicate if changes were made. The Creative Commons Public Domain Dedication waiver (http://creativecommons.org/publicdomain/zero/1.0/) applies to the data made available in this article, unless otherwise stated. 
Synovial MSCs have a higher chondrogenic potential than bone marrow MSCs [11]. Transplantation of synovial MSCs regenerated cartilage [12] and meniscus [13]. Synovial MSCs are clinically used for cartilage regeneration [14]. To prepare synovial MSCs, synovium is digested, and unfractionated synovial cells are expanded to form cell colonies of synovial MSCs $[15,16]$. Synovial tissue can be histologically classified into three regions; surface, stromal, and perivascular regions [17]. If synovial cells can be obtained and synovial MSCs can be prepared from each region separately, more attractive synovial MSCs can be used in clinical therapies. This also provides important information on the physiological roles of cells in the synovium. The purpose of the present study was to identify specific markers for the isolation of synovial cells in the surface, stromal, and perivascular regions, and to compare properties of MSCs sorted by the specific markers.

\section{Methods}

\section{Human synovium}

This study was approved by the Medical Research Ethics Committee of Tokyo Medical and Dental University and all human study subjects provided informed consent. Human synovium was harvested from the knees of ten donors (59-85 years) with osteoarthritis during total knee arthroplasty.

\section{Transmission electron microscopy (TEM)}

The specimens of synovial tissues were rapidly fixed in $2.5 \%$ glutaraldehyde in $0.1 \mathrm{M}$ phosphate buffer for $2 \mathrm{~h}$. The samples were washed with $0.1 \mathrm{M}$ phosphate buffer, post-fixed in $1 \% \mathrm{OsO}_{4}$ buffered with $0.1 \mathrm{M}$ phosphate buffer for $2 \mathrm{~h}$, dehydrated in a graded series of ethanol and embedded in Epon 812. Ultrathin sections at $90 \mathrm{~nm}$ were collected on copper grids, double-stained with uranyl acetate and lead citrate, and then examined by transmission electron microscopy (H-7100, Hitachi, Tokyo, Japan) [18].

\section{Immunostaining}

Synovial tissues were rapidly embedded in OCT compound (Sakura Finetec Japan, Tokyo, Japan) and 4\% carboxymethyl cellulose and were washed with $0.1 \%$ Tween-TBS. After blocking with Protein Block SerumFree (Dako, Glostrup, Denmark), sections (5 $\mu \mathrm{m}$ thick) were incubated with 19 antibodies; CD90 (Becton, Dickinson and Company; BD, Franklin Lakes, NJ, USA), CD44 (BD), CD73 (BD), CD105 (BD), CD271 (Miltenyi Biotec, Bergisch Gladbach, Germany), CD140a (BD), CD140b (BD), CD29 (Merck Millipore, Darmstadt, Germany), CD49f (Merck Millipore), Ki67 (Dako), Proliferating Cell Nuclear Antigen (PCNA; Santa Cruz Biotechnology, Inc., Santa Cruz, CA, USA), CD55
(Miltenyi Biotec), CD31 (antibody derived from mouse (Dako) for IHC and sheep (R\&D Systems, Minneapolis, MN, USA) for IF), CD146(BD), Laminin (Dako), Collagen type IV (Dako), Proteoglycan 4/Lubricin (PRG4; Santa Cruz Biotechnology), Hyaluronan synthase 1 (HAS-1; Santa Cruz Biotechnology) and HAS-2 (Santa Cruz Biotechnology), at $4{ }^{\circ} \mathrm{C}$ overnight. After washing three times, secondary antibodies (Chemmate Envision HRP-polymer, Dako) or anti-goat horseradish peroxidase (HRP)-conjugated secondary antibody (Dako) were added, followed by incubation for $30 \mathrm{~min}$ at room temparature. Staining was simultaneously developed in DAB + solution (Dako), with counterstaining by hematoxylin. Samples were analyzed with a light microscope. The $\mathrm{DAB}$ positive intensity of surface and matrix protein expression in synovium was quantified with Image J software after immunostaining [19].

For fluorescence microscopy images, sections were first washed then incubated with Alexa Fluor 488- and/ or 594-conjugated secondary antibodies (1:500; Thermo Fisher Scientific, Waltham, MA, USA) specific for the appropriate species for $1 \mathrm{~h}$ at room temperature. Samples were counterstained with 4,6-diamidino-2-phenylindole (DAPI; Wako, Osaka, Japan) and analyzed with a microscope (BZ-X700, Keyence Co., Ltd., Osaka, Japan).

\section{Flow cytometric isolation and analysis}

Synovium was digested in a solution of $3 \mathrm{mg} / \mathrm{mL}$ collagenase (Sigma-Aldrich Japan, Tokyo, Japan) at $37^{\circ} \mathrm{C}$. After $3 \mathrm{~h}$, the digested cells were filtered through a $70-\mu \mathrm{m}$ cell strainer (Greiner Bio-One GmbH, Kremsmunster, Austria). The cells from six donors were harvested using a cell-dissociation buffer. Cells were suspended in HBSS at a density of $5 \times 10^{5}$ cells/mL and stained for $30 \mathrm{~min}$ on ice with the antibodies. For cell isolation, cells were stained with CD31-PE-Cy7 (BD), CD45-PE-Cy7 (BD), CD235a-PE-Cy7 (BD), CD55-FITC (Miltenyi Biotec), CD90-PE (BD) and CD271-APC (Miltenyi Biotec) were used at day 0. Flow cytometric isolation of cell surface antigens were performed by a double-laser Aria 2 system (BD). For cell surface analysis, cells were stained with CD31-FITC (BD), CD45-FITC (BD), CD44-APC-H7 (BD), CD73-BV421 (BD), CD90-PE (BD), CD105-PerCP-Cy5.5 (BD), CD55-PE (BD), CD271-APC (Miltenyi Biotec), CD140b-PerCP-Cy5.5 (BD) and CD146-FITC (BD) at passage 3. Flow cytometric analysis of cell surface antigens was performed by a triple-laser FACS Verse system (BD). These data were analyzed using FlowJo software (Tree Star Inc., Ashland, OR, USA). Flow cytometric analyses were also performed for expanded cells at passage 3 .

\section{Colony formation and proliferation ability of synovial MSCs}

For proliferation assays, bulk and sorted synovial cells from four donors were plated on six wells at 2000 cells 
per $10 \mathrm{~cm}^{2}$ wells for 10 days in complete culture medium with 10\% FBS (Thermo Fisher Scientific, Inc.) and 1\% penicillin/streptomycin in alpha MEM (Thermo Fisher Scientific, Inc.). Cultured cells were harvested with $0.25 \%$ trypsin and $1 \mathrm{mM}$ ethylenediaminetetraacetic acid (EDTA) (Thermo Fisher Scientific, Inc) at $37{ }^{\circ} \mathrm{C}$ for $5 \mathrm{~min}$ and counted with cell-counting plates. For colony formation assays, bulk and sorted synovial cells from four donors were plated as above. The dishes were stained with crystal violet at 14 days and the colony number was counted.

\section{Differentiation assay of synovial MSCs}

For chondrogenic differentiation, cultured synovial MSCs from four donors at passage 2 were harvested using a celldissociation buffer as time 0 or cells at $48 \mathrm{~h}$ were harvested from preservation tubes after preservation. Then 2 . $5 \times 10^{5}$ cells were transferred to a $15 \mathrm{~mL}$ tube (BD Falcon) and cultured in chondrogenic induction medium containing $10 \mathrm{ng} / \mathrm{mL}$ transforming growth factor- $\beta 3$ (Miltenyi Biotec) and $1 \mu \mathrm{g} / \mathrm{mL}$ bone morphogenetic protein 2 (Medtronic, Minneapolis, MN, USA), in high glucose DMEM with $1 \%$ antibiotic-antimycotic (Thermo Fisher Scienific) which was changed every 3-4 days. After 21 days, chondrogenic differentiated cells were analyzed by safranin-o (Wako) staining.

For calcification induction, 100 cells were transferred to a $60 \mathrm{~cm}^{2}$ dish and cultured for 14 days in complete culture medium. Adherent cells were then cultured in osteogenic induction medium containing $50 \mu \mathrm{g} / \mathrm{mL}$ ascorbic acid 2-phosphate (Wako), $10 \mathrm{nM}$ dexamethasone (Wako), and $10 \mathrm{mM} \beta$-glycerophosphate (SigmaAldrich), in complete culture medium, which was changed every 3-4 days. After 21 days, the differentiation of these cells into osteoblasts was assessed by alizarin red staining (Merck Millipore). To quantify the amount of alizarin red, the deposition was extracted by $10 \%(\mathrm{w} / \mathrm{v})$ cetylpyridinium chloride (Sigma-Aldrich) in $10 \mathrm{mM}$ sodium phosphate $(\mathrm{pH} \mathrm{7.0)}$ at room temperature for $1 \mathrm{~h}$ and the alizarin red stain in the extraction buffer was determined by measuring the optical density of the solution at $560 \mathrm{~nm}$ absorbance [15].

For adipogenic differentiation, adherent cells were cultured in adipogenic induction medium (Lonza, Basel, Switzerland), which was changed every 3-4 days. After 21 days, oil red-o staining (Muto Pure Chemicals, Tokyo, Japan) confirmed the differentiation of these cells into adipocytes. To quantify adipogenic ability, the amount of triglyceride was measured by adipogenesis assay kit (BioVision, Milpitas, CA, USA) in accordance with manufacturer's instructions.

\section{RNA isolation and RT-PCR analysis}

For chondrogenesis, six pellets from each donor were digested together. For calcification and adipogenesis,
MSCs were plated at 50 cells $/ \mathrm{cm}^{2}$ in $145 \mathrm{~cm} 2$ plates and extracted from two dishes. Total RNA was extracted using RNeasy Mini Kit (Qiagen N.V., Venlo, Netherlands). Concentration and quality of the RNA were verified on a Quantus Fluorometer (Promega Co., Madison, WI, USA). The cDNA was synthesized with random hexamer primers from total RNA using the Transcriptor High Fidelity cDNA Synthesis kit (Roche Diagnostics, Basel, Switzerland). Real-time PCR was performed in a LightCycler 480 instrument (Roche Diagnostics). PCR reaction used the LightCycler 480 Probes Master. Relative amounts of mRNA were calculated and standardized as previously described [20]. The following TaqMan gene expression assay kits (Integrated DNA Technologies, IA, USA) were used as Hs.PT.39a. 22214847 for $A C T B$, Hs.PT.58.38984663 for SOX9, Hs. PT.58.38672730 for COL10A1, Hs.PT.56a.742783 for $A C A N$, Hs.PT.56a.40555206 for ALP, Hs.PT.56a. 19568141 for RUNX2, Hs.PT.58.25464465 for PPARG, Hs.PT.58.4022335.g for CEBPA, Hs.PT.58.3040231 for GTF3A, and Hs.PT.58.20087469 for LPL.

\section{Statistical analysis}

All data were statistically evaluated with GraphPad Prism 6 (GraphPad Software, La Jolla, CA, USA). Data are expressed as mean $\pm \mathrm{SD}$. Each statistical analysis method is described in the legend. Two-tailed $P$ values of $<0.05$ were considered to be significant.

\section{Results}

TEM images for surface, stromal, and perivascular regions of synovium

Synovium can be histologically classified into three regions; surface, stroma, and perivascular regions (Fig. 1a). These three regions had different ultra-microstructures. The surface region of the synovial membrane primarily consisted of macrophage and fibroblast cell components (Fig. 1b). The stromal region, defined as subsynovial tissue excluding the perivascular region, consisted of stromal cells with collagen fibrils. The perivascular region in synovial tissue contained blood vessels with perivascular cells.

\section{Immunological characterization for surface, stromal, and perivascular region of synovium}

To characterize the three regions immunologically, immunostaining with 19 antibodies was performed. We were able to detect the following proteins in the surface, stroma or perivascular region; MSC markers (CD90, CD44 and CD73), growth factor receptors of MSC markers (CD105, CD271, CD140a and CD140b), integrins (CD29 and CD49f), proliferative markers (Ki67, PCNA), complement inhibitor (CD55), endothelial 


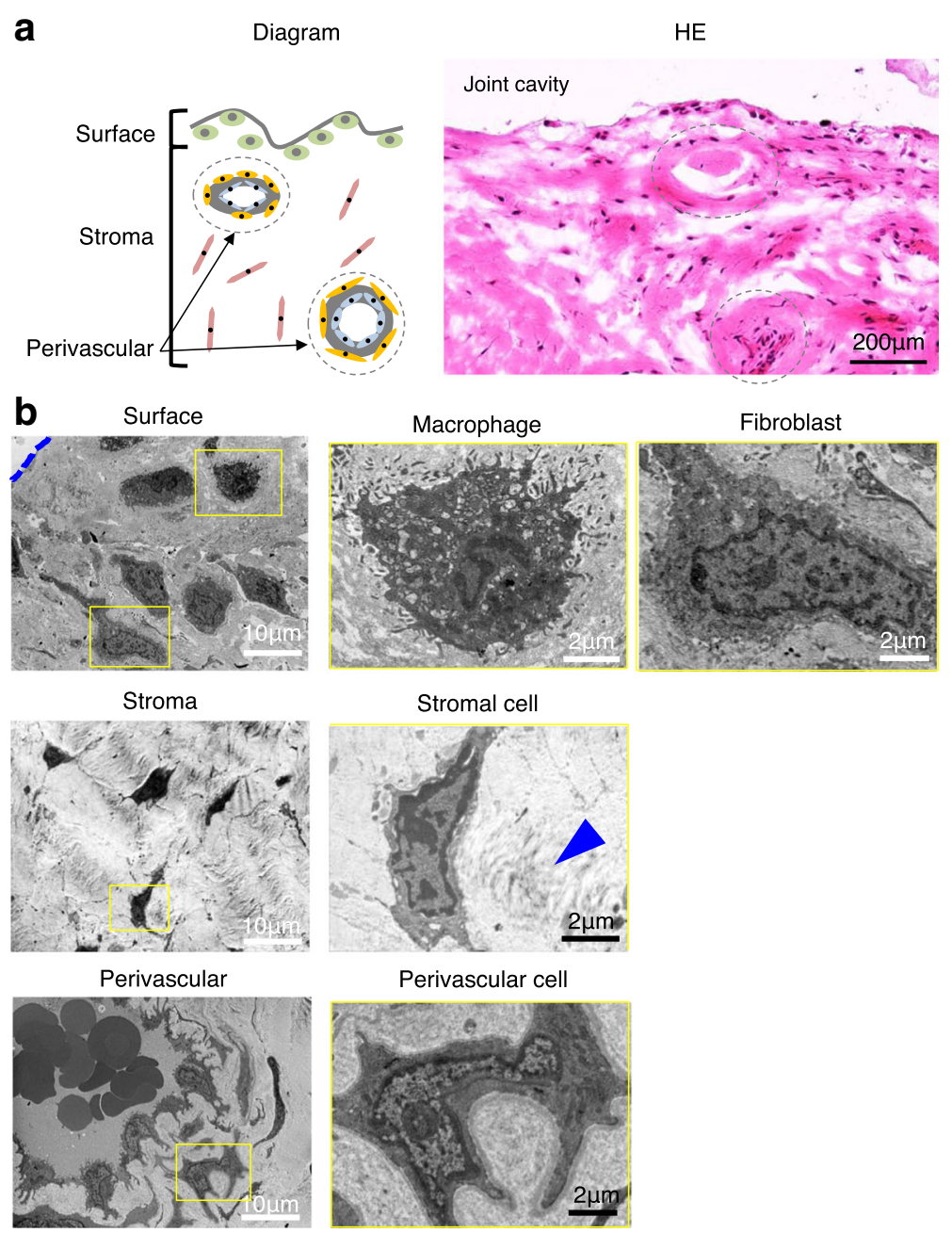

Fig. 1 Surface, stromal, and perivascular regions of human synovium. a Diagram and H\&E-stained histological image of synovium. b Transmission electron microscope (TEM) images. Surface of the synovium is indicated by dashed line. Around a stromal cell, collagen fibrils are indicated by an arrowhead

markers (CD31 and CD146), and extracellular matrix (Laminin, Col4, PRG4, HAS-1 and HAS-2) (Fig. 2).

The expression of proteins was region specific. Generally, in the surface region, MSC markers, proliferation markers, complement inhibitor, and extracellular matrices were highly expressed. In the stromal region, only MSC markers were highly expressed. In the perivascular region, most proteins we examined, with the exception of complement inhibitor, were highly expressed (Fig. 3a).

\section{Specific markers for isolation of synovial cells from surface, stromal, and perivascular regions}

As specific markers for isolation of synovial cells from each region, we selected CD55 as a positive marker for synovial cells derived from the surface region, CD271 and CD55 as negative markers for synovial cells derived from the stromal region, and CD271 as a positive marker for synovial cells derived from the perivascular region (Fig. 3a). We confirmed the two markers by fluorescent immunostaining (Fig. 3b). $\mathrm{CD} 55^{+}$cells were found in the surface region and were colocalized with basement membrane positive for laminin. CD55 was also expressed on endothelial cells, but the level was lower than that of the surface region cells. CD271 ${ }^{+}$cells were confirmed in perivascular cells positive for CD31, while CD271 was not expressed in stromal cells negative for CD31.

\section{Ratio of synovial cells in the three regions by flowcytometric isolation}

From the non-hematopoietic lineage cells, synovial cells in the surface regions were sorted for CD55, those in the stromal region were negatively sorted for CD55 and CD271, and those in the perivascular region were sorted for CD271 (Fig. 4a). The ratio of the gated cells to $\mathrm{PI}^{-}$ cells was $25 \%$ in the hematopoietic cells and $70 \%$ in the 


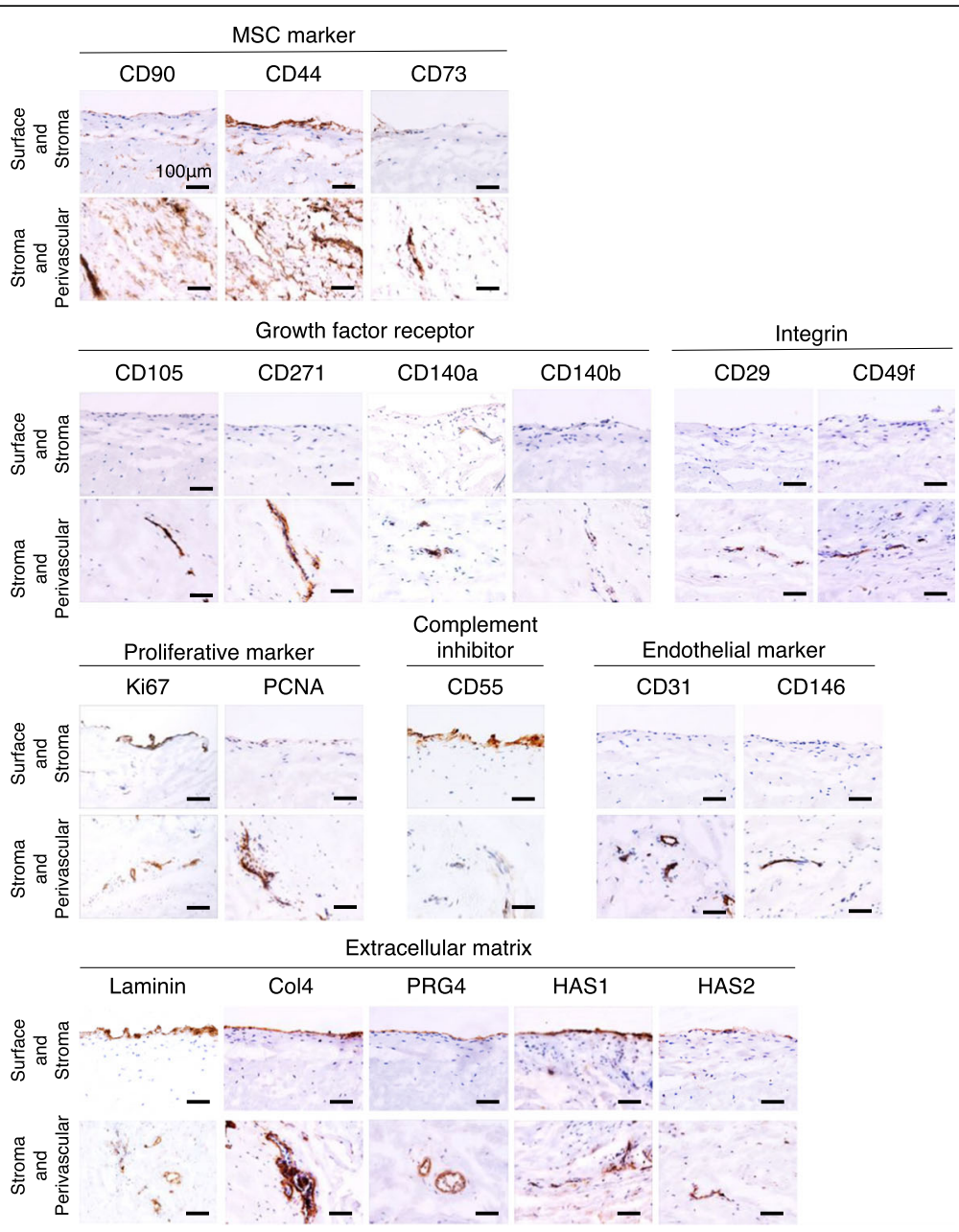

Fig. 2 Immunostained images for surface, stromal, and perivascular regions of human synovium

non-hematopoietic cells (Fig. 4b). The ratio of the sorted cells to other non-hematopoietic lineage cells was approximately $5 \%$ in the surface region, $70 \%$ in the stromal region, and $15 \%$ in the perivascular region (Fig. 4c). The ratio of synovial cells derived from the stroma region was statistically higher than from the surface or perivascular regions.

\section{Proliferation and colony formation in the bulk and sorted synovial cells}

We prepared five fractions of synovial cells; the bulk fraction, the non-hematopoietic and endothelial fraction, the surface fraction, the stromal fraction, and the perivascular fraction. No obvious morphological differences were observed among the five fractions (Fig. 5a). Proliferation was the highest in the perivascular fraction (Fig. 5b). Colony morphology appeared similar and no significant differences in colony number were obtained among the five fractions (Fig. $5 \mathrm{c}$ and $\mathrm{d}$ ).
Surface markers of colony-forming cells derived from the bulk and sorted synovial cells

Passage 3 colony-forming cells expressed CD44, CD73, and CD90 at high rates (over 90\%); CD55 and CD105 at a moderate rate (50-90\%); CD140b and CD271 at low rates (approximately 10\%); and did not express CD31, CD45, and CD146 (less than 3\%) (Fig. 5e). This expression pattern was similar to MSCs. Though synovial cells in the perivascular fraction were obtained after sorting with CD 271, the positive rate decreased to only $5 \%$ after colony formation.

\section{Differentiation of colony-forming cells derived from the bulk and sorted synovial cells}

After condrogenic induction, cell pellets from the five fractions were differentiated into cartilage that stained positive for safranin-o (Fig. 6a). The diameter of the cartilage pellets was the largest in the perivascular fraction among the five fractions (Fig. 6b). The perivascular fraction showed higher mRNA expression of SOX9, 


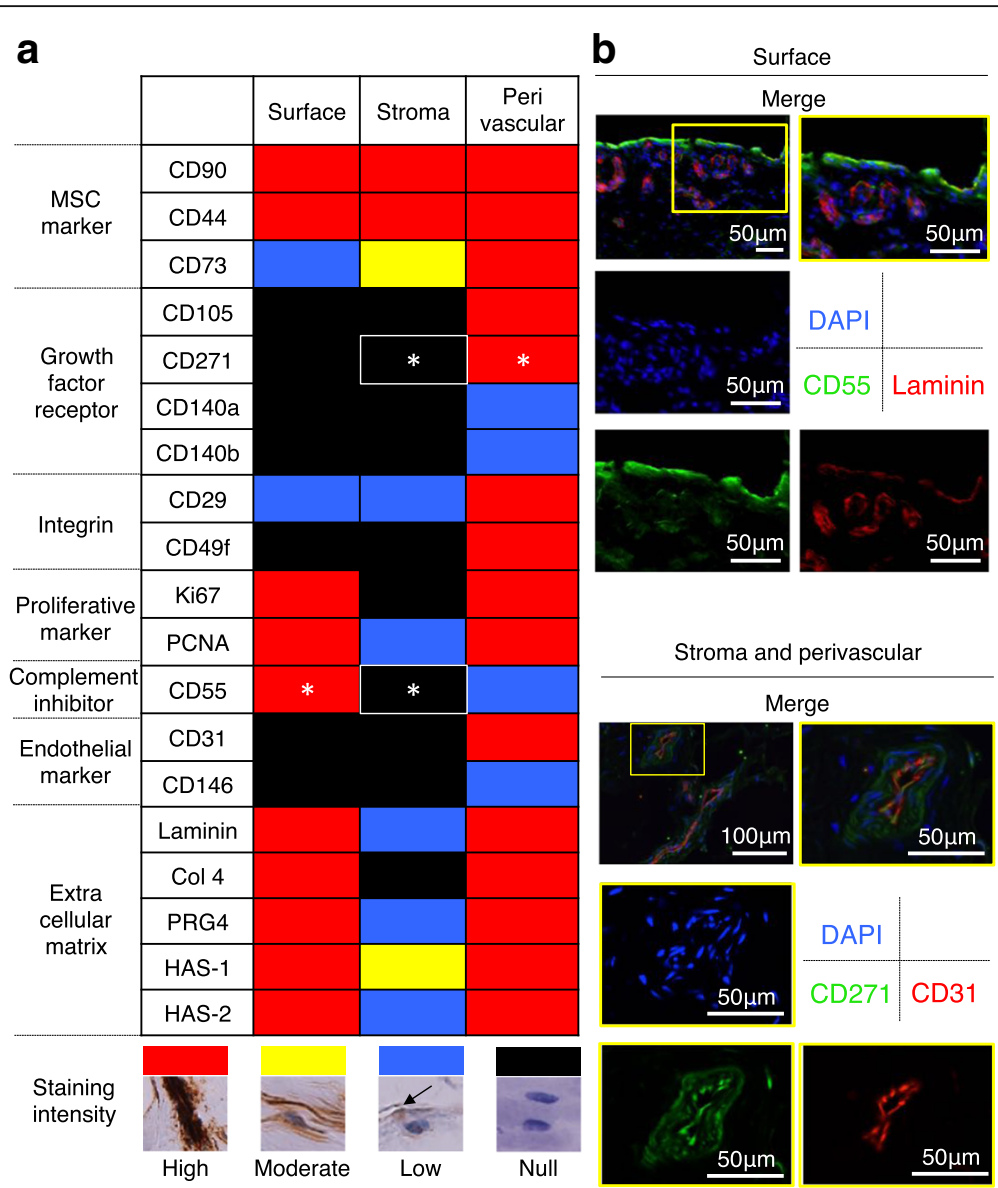

Fig. 3 Immunological characterization for surface, stromal, and perivascular regions of human synovium. a Summary of immunological analysis for surface, stromal, and perivascular regions of human synovium. The staining intensity was subjectively graded into four colours; high (red), moderate (yellow), low (blue), and null (black) based on immunostained synovium derived from six patients. *; specific markers we selected for isolation of synovial cells derived from each region. b Fluorescent microscope images for synovium immunostained with specific markers for isolation of synovial cells derived from each region

Aggrecan $(A C A N)$, and COL10A1 in the cartilage pellets (Fig. 6c).

After calcification induction, the colony-forming cells derived from synovial cells stained positive for alizarin red in the five fractions (Fig. 6c). Absorbance of alizarin red, used to quantify differentiation was also the highest in the perivascular fraction (Fig. 6d). Real-time RT-PCR analyses showed similar expression levels of $A L P$ and RUNX2 for the calcification observed for each region (Fig. 6f). No expression of osteogenic genes, such as $O P N, O C N, O S X$, and DLX5, was detected (data not shown).

After adipogenic induction, colony-forming cells derived from synovial cells stained positive for oil red-o in the five fractions (Fig. 6e). No significant differences were observed in triglyceride production among the five fractions (Fig. 6f). Real-time RT-PCR analyses showed similar expression levels of PPARG and $L P L$ for the adipogenesis derived from each region (Fig. 6i). GTF3A and
$C E B P A$ expression was lower for the surface fraction than for the other four regions.

\section{Discussion}

We used histological evaluations to classify the synovium into three regions: the surface, stroma, and perivascular regions. We then characterized the three regions immunologically by immunostaining with 19 antibodies. Based on the results, we selected CD55 as a positive marker for synovial cells derived from the surface region, CD271 and CD55 as negative markers for synovial cells derived from the stromal region, and CD271 as a positive marker for synovial cells derived from the perivascular region. The number of synovial cells derived from the perivascular region was much smaller than that derived from the stromal region, but the colony-forming cells derived from the perivascular region had higher proliferative and chondrogenic potentials. 


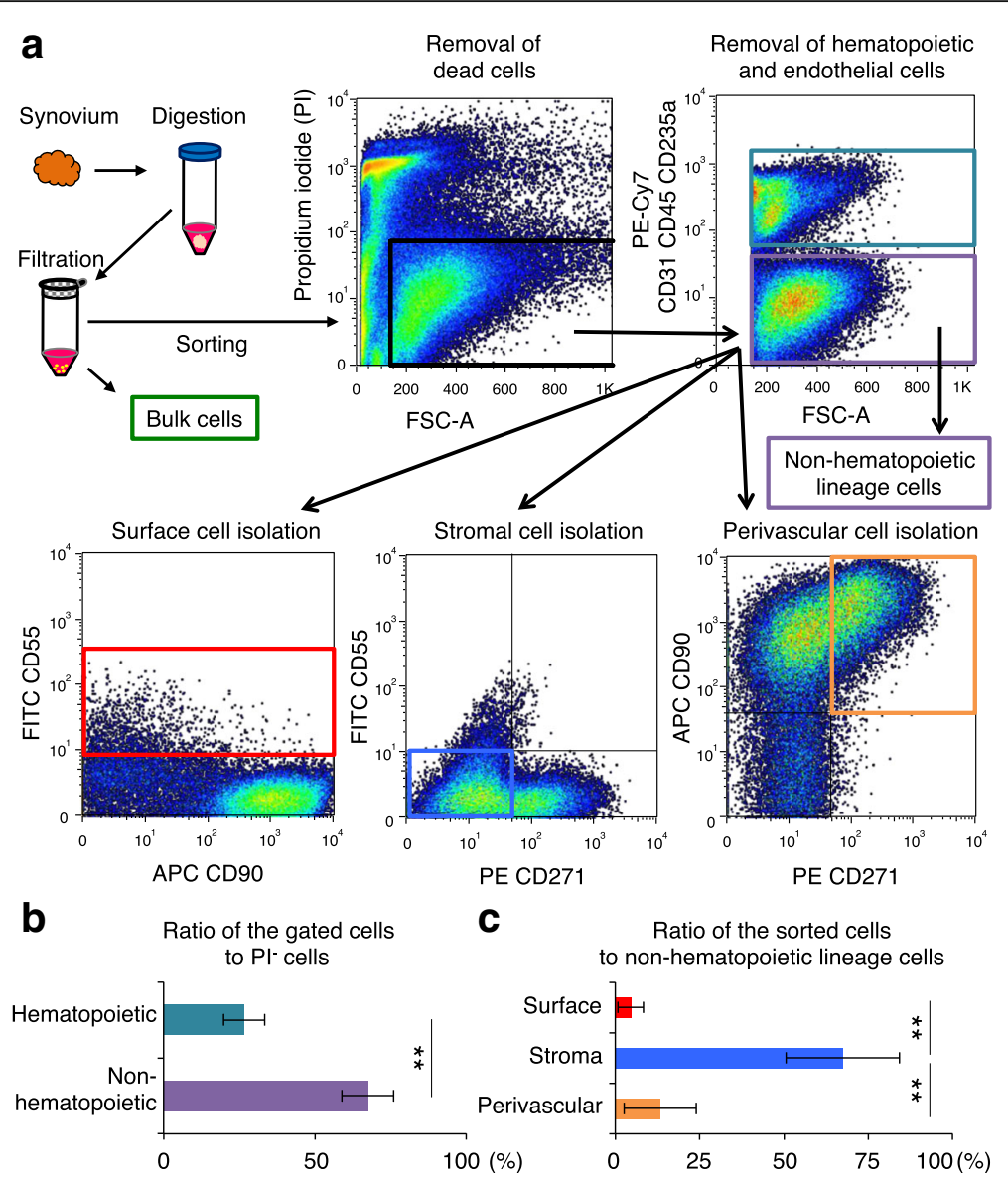

Fig. 4 Ratio of the sorted surface, stromal and perivasucular cells. a Scheme for isolating surface, stromal and perivasucular cells. Synovium was digested and filtrated for bulk cells. From the bulk cells, dead cells were removed, then hematopoietic and endothelial cells were removed for non-hematopoietic lineage cells. From the non-hematopoietic lineage cells, surface cells were sorted for CD55, stromal cells were negative sorted for CD55 and CD271, and perivascular cells were sorted for CD90 and CD271. Non-hematopoietic lineage cells consisted of Pl ${ }^{-} \mathrm{CD}_{1} 1^{-} \mathrm{CD} 45^{-}$ CD235a ${ }^{-}$cells. $\mathbf{b}$ Ratio of the gated cells to $\mathrm{Pl}^{-}$cells. Bar shows mean \pm SD. The analyzed synovium were obtained from eight donors were analyzed. ${ }^{* *} p<0.01$ by Wilcoxon matched-pairs signed rank test. c Ratio of the sorted cells to non-hematopoietic lineage cells. Bar shows mean \pm SD. Synovia derived from eight donors were analyzed. ${ }^{*} p<0.01$ by the Friedman test followed by Steel-Dwass multiple comparisons

We selected CD55, a complement inhibitor, as a specific marker for the surface region because activation of complement in the synovial membrane plays an important role in the pathogenesis of osteoarthritis [21]. CD55 is also recognized as a decay-accelerating factor (DAF) and is expressed in the synovial lining during inflammation. Synovial tissues derived from patients with osteoarthritis are exposed to inflammatory conditions in the knee joint, so the surface region is in constant contact with synovial fluid containing inflammatory cytokines. Analysis of MSCs positive for CD55 will therefore provide a better clarification of the pathology of osteoarthritis.

We selected CD271 as a specific marker for the perivascular region. CD271 is a low-affinity nerve growth factor receptor (LNGFR) and serves as one of the two receptor types for neurotrophins, a family of protein growth factors that stimulate the survival and differentiation of neuronal cells. These nerve cells in the intraarticular tissues are thought to be important in cartilage repair after cartilage damage, as demonstrated in mouse studies [22]. CD271 also serves as a marker of MSCs with high colony-forming ability in bone marrow and synovium [23-25]. The role of CD271 in the perivascular region of the synovium is unknown, but CD271 expression is possibly related to the pathophysiological response of vascular developments/neurogenesis associated with the synovitis of osteoarthritis. Functional analysis CD271 expression by the CD271 $1^{+}$cells in the synovial tissues will contribute to a greater understanding of the healthy and diseased state.

Though synovial cells in the perivascular fraction were obtained after sorting with CD 271, the positive rate decreased to only $5 \%$ after colony formation. This means that positive rate of CD271 decrease after expanding of the sorted cells. There are some reports describing that positive rate of CD271 decreased after expanding of 

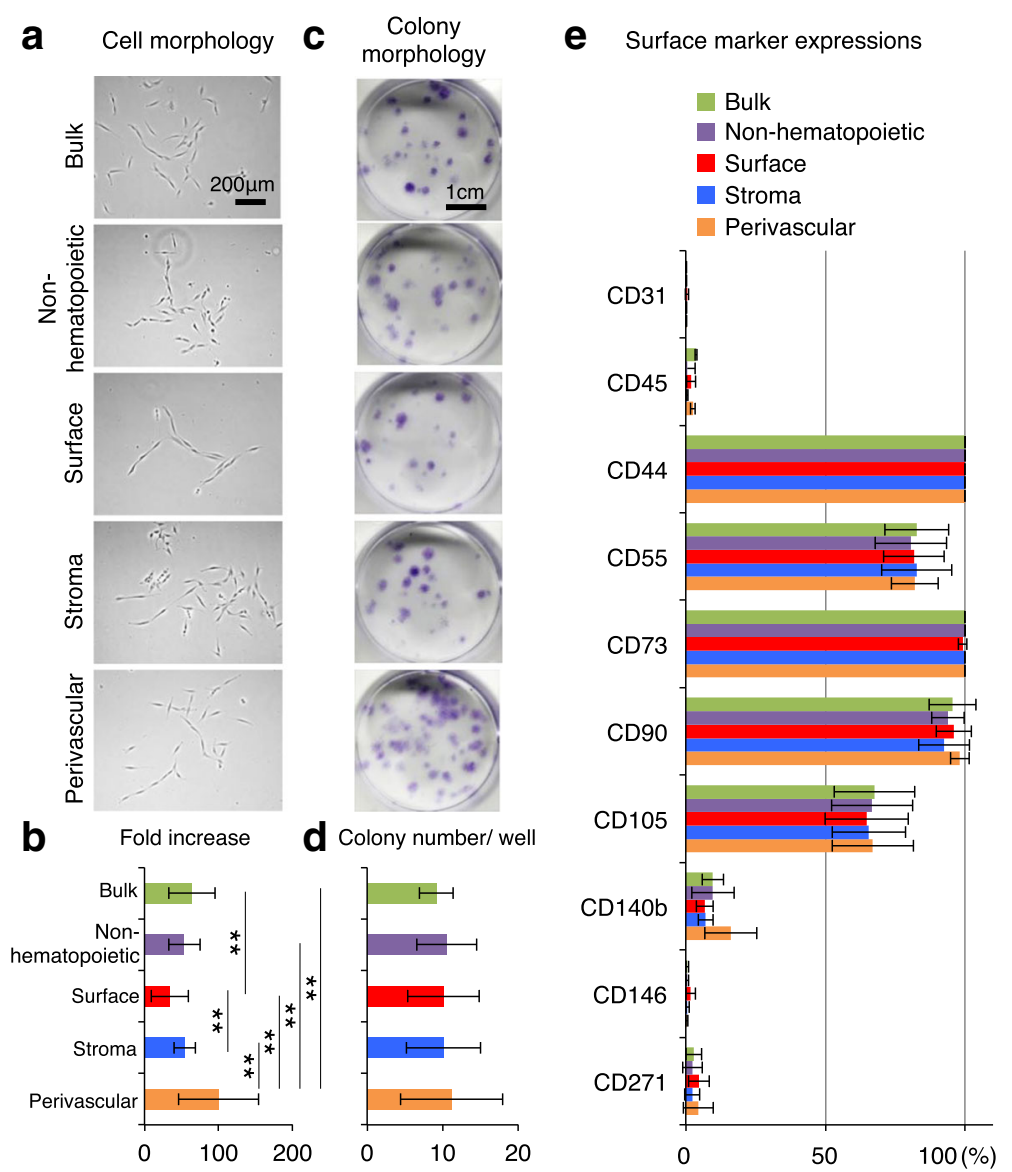

Fig. 5 Proliferation and surface marker expression of colony-forming cells derived from non-sorted and sorted synovial cells. a Cell morphology of synovial cells 5 days after plating. $\mathbf{b}$ Fold increase of synovial cells. Bulk and sorted cells were plated at 200 cells/ $\mathrm{cm}^{2}$ and cultured for 10 days. Bar shows mean $\pm \mathrm{SD}$. ${ }^{* *} p<0.01$ by the Friedman test followed by Steel-Dwass multiple comparisons (four donors, $n=6$ for each donor). $\mathbf{c}$ Colony morphology of synovial cells. Bulk and sorted cells were plated on six-well plates at 100 cells/cm², cultured for 14 days and stained with crystal violet. $\mathbf{d}$ Colony number/well. Bar shows mean \pm SD (four donors $n=6$ for each donor). e Surface markers of cultured MSCs. Bar shows mean \pm SD (four donors)

CD271 ${ }^{+}$MSCs $[23,26]$. Although the reason for the reduction of CD271 expression is not clear, one possibility is that the alteration of the environment from in vivo three-dimensional surroundings, in which $C D$ 271-positive cells located at synovial perivascular region, to in vitro two-dimensional surroundings, in which $C D$ 271-positive cells were expanded on culture dishes.

We selected CD55 and CD271 as negative specific markers for the stroma region, as our initial attempts to identify positive markers specific for the stroma region were unsuccessful. Other negative markers specific for the stroma region were CD55 (complement inhibitor), Ki67, and Col4 (extracellular matrix marker).

The surface marker expression pattern in the colonyforming cells derived from the bulk and sorted synovial cells in each group was similar to that observed for MSCs. These colony-forming cells also demonstrated multi-potentiality, indicating that indicate they were MSCs. This finding further confirmed that MSCs can be obtained from synovium, irrespective of sorting. Even without sorting, a small number of hematopoieticlineage cells adhere to plastic dishes, and the rate of adhesion of hematopoietic-lineage cells further decreases after colony formation of the non-hematopoietic cells.

The MSCs derived from the perivascular fraction showed the largest cartilage pellets and the highest expression levels of SOX9 and Aggrecan mRNA during chondrogenesis. The pellet size reflects the chondrogenic potential for each population of MSCs, whereas SOX9 and Aggrecan mRNA expression reflects the chondrogenic potential of each individual MSC. Thus, the MSCs derived from the perivascular fraction had the highest chondrogenic potential, whether expressed per population or per single cell. However, these MSCs also showed the highest COL10A1 mRNA expression, suggesting that their potential for hypertrophic chondrocyte differentiation though this was not evident in histological analyses. 


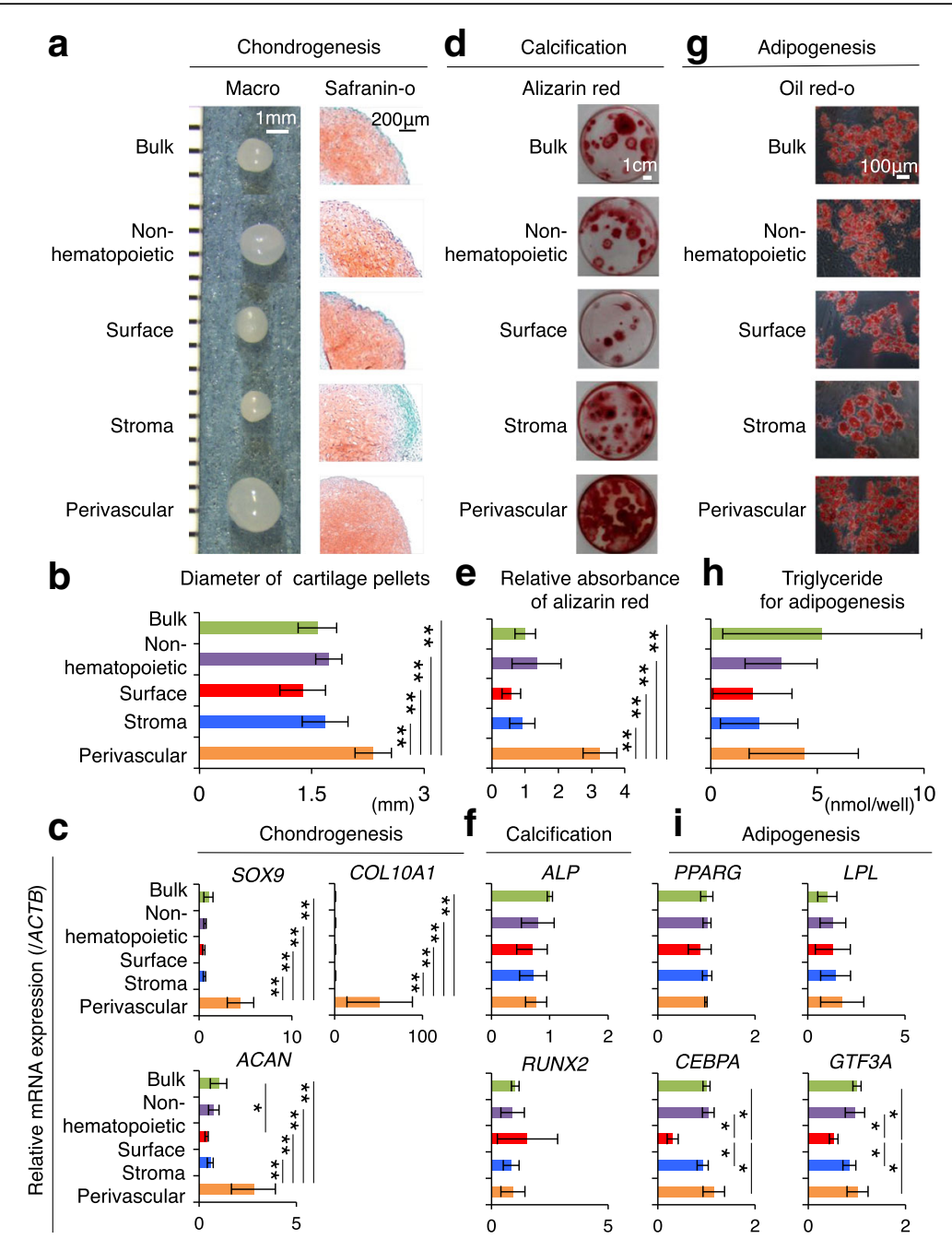

Fig. 6 Differentiation ability of colony-forming cells derived from non-sorted and sorted synovial cells. a Chondrogenesis. Macroscopic images and histological sections stained with safranin-o are shown. $\mathbf{b}$ Diameter of cartilage pellets. Bar shows mean \pm SD. ${ }^{* *} p<0.01$ by the Kruskal-Wallis test followed by Dunn multiple comparisons (four donors, $n=4$ for each donor). c Relative mRNA expression per SOX9, Aggrecan (ACAN), and COL10A1 by real-time RT-PCR analyses using $\beta$-actin (ACTB) as housekeeping gene. Gene expression fold changes are shown and normalized to bulk as 1. Bar shows mean $\pm \mathrm{SD}$ (three donors, $\mathrm{n}=4$ for each donor). ${ }^{* *} p<0.01$ by the Friedman test followed by Steel-Dwass multiple comparisons. $\mathbf{d}$ Calcification. Culture dishes stained with alizarin red are shown. e Quantification of alizarin red staining for calcification. Absorbance of alizarin red in the bulk group was set as 1 . Bar shows mean \pm SD. ${ }^{* *} p<0.01$ and ${ }^{*} p<0.05$ by the Kruskal-Wallis test followed by Dunn multiple comparisons (four donors, $n=4$ for each donor). $\mathbf{f}$ Relative mRNA expression per ALP and RUNX2 by real-time RT-PCR analyses using $\beta$-actin (ACTB) as housekeeping gene. Gene expression fold changes are shown and normalized to bulk as 1. Bar shows mean \pm SD (three donors, $n=2$ for each donor). $\mathbf{g}$ Adipogenesis. Culture dishes stained with oil red-o are shown. $\mathbf{h}$ Quantification of triglyceride for adipogenesis. Bar shows mean \pm SD (four donors, $\mathrm{n}=4$ for each donor). $\mathbf{i}$ Relative mRNA expression per PPARG, LPL, GTF3A, and CEBPA by real-time RT-PCR analyses using $\beta$-actin (ACTB) as housekeeping gene. Gene expression fold changes are shown and normalized to bulk as 1 . Bar shows mean $\pm \mathrm{SD}$ (three donors, $\mathrm{n}=2$ for each donor). ${ }^{*} p<0.05$ by the Friedman test followed by Steel-Dwass multiple comparisons

The perivascular fraction also showed the highest absorbance of alizarin red, indicating pronounced calcification. This high absorbance might reflect the high number of cells present just before calcification induction, because MSCs derived from the perivascular fraction had a high proliferation capability. By contrast, the other five fractions showed equivalent mRNA expression of $A L P$ and RUNX2 and no mRNA expression of other osteogenic genes, such as DLX5, OSX, OPN, and OCN (data not shown). Based on these findings, we assume that this differentiation assay does not mimic the osteogenesis of MSCs, as we mentioned previously [20]. For this reason, we have used the term "calcification" rather than "osteogenesis" [27, 28].

The five fractions also showed equivalent triglyceride production and mRNA expression of PPARG and $L P L$, indicating similar adipogenesis activity. Conversely, mRNA expression levels of GTF3A and CEBPA were 
higher in the surface fraction than in the other fractions. PPARG and CEBPA are early markers and LPL and GTF3A are late markers of adipogenesis, so these contradictory expression patterns cannot be explained by different developmental stages. They may possibly reflect the complexity of molecular regulation of adipogenesis [29].

The perivascular fraction contained MSCs that were superior in proliferation and cartilage differentiation when compared to the MSCs in the other fractions. Caplan et al. recently advocated that MSCs are derived from pericytes, and our results support this hypothesis [30]. However, our results also demonstrated that MSCs are present in the surface and stromal regions, as well as in the perivascular region, although the MSCs from the surface and stromal regions showed poorer potentials for proliferation and cartilage differentiation.

The MSCs from the three synovial regions showed similar expression patterns of ten surface markers after expansion, but demonstrated different patterns of proliferation and differentiation. One reason for these differences might be that the three populations are still distinct, even though they show similar expression patterns for the ten surface markers. Other surface markers will therefore be useful to further distinguish these three populations. For example, Mabuchi et al. reported that the $\mathrm{CD} 90^{+}$and $\mathrm{CD} 271^{+}$fractions of bone marrow MSCs differed in their proliferation ability with and without expression of CD106 and CD49d [23]. Identification of surface markers or transcription factors to distinguish differentiation potentials among the three groups will be important for clarifying synovial MSC biology.

A higher proliferation and chondrogenic potential was observed in the present study MSCs derived from the perivascular region, although the number of sorted cells in the perivascular region was only approximately $20 \%$ of that in the stromal region. The MSCs derived from the stromal region showed poorer chondrogenic potentials; however, because greater numbers of MSCs could be prepared from that region than from the perivascular region, these differences in chondrogenic potential might be minimized. Therefore, from the standpoint of total cells harvested, the synovial stroma region could be a suitable MSC source.

The current study has three limitations that should be considered. First, the staining for the immunological characterization of surface, stromal, and perivascular regions of human synovium was graded subjectively and not objectively. Second, we selected $\mathrm{CD} 55^{+}$as the marker for synovial cells from the surface region, but because we investigated synovium derived from osteoarthritis patients, these cells may be representative of the inflammatory condition. Third, in vivo chondrogenesis was not evaluated.

\section{Conclusions}

In conclusion, we selected CD55 as a positive marker for synovial cells derived from the surface region, CD271 and CD55 as negative markers for synovial cells derived from the stromal regions, and CD271 as a positive marker for synovial cells derived from the perivascular region. The number of synovial cells derived from the perivascular region was much lower than that derived from the stromal region, but the colony-forming cells derived from the perivascular region had higher proliferative and chondrogenic potentials.

\section{Acknowledgements}

We would like to thank Ms. Mika Watanabe and Ms. Kimiko Takanashi for the management of our laboratory and Ms. Ellen Roider for English editing. Yuji Kohno, Yusuke Nakagawa, Tomomasa Nakamura, Masafumi Horie, and

Toshifumi Watanabe contributed to the recruitment of the patients and the acquisition of the written informed consent from the patients.

\section{Funding}

This study was supported by the Japan Society for the Promotion of Science (JSPS) to MM (16H06262) and by the Highway Program for Realization of Regenerative Medicine from the Japan Agency for Medical Research and Development (AMED) to IS (JP16bm0504001).

\section{Availability of data and materials}

All the data supporting the results can be found in this manuscript and supplemental data. Please contact the corresponding author for more data requests.

\section{Authors' contributions}

$\mathrm{MM}$ and IS contributed to study conception and design. MM, YM, YO, SI, KK, and SF contributed to acquisition of data. KO, NO, HKa, HKo, KT, CA, and TM contributed to analysis and interpretation of data. All authors were involved in drafting the article or revising it critically for important intellectual content, and all authors approved the final version to be published. IS had full access to all of the data in the study and takes responsibility for the integrity of the data and the accuracy of the data analysis.

\section{Ethics approval and consent to participate}

This study was properly approved and certificated to be in compliance with the Helsinki Declaration by the institutional review board of Tokyo Medical and Dental University (reference number: M2000-2121). Written informed consent forms were submitted by all the participating patients.

\section{Competing interests}

The authors declare that they have no competing interests.

\section{Publisher's Note}

Springer Nature remains neutral with regard to jurisdictional claims in published maps and institutional affiliations.

\section{Author details}

${ }^{1}$ Center for Stem Cell and Regenerative Medicine, Tokyo Medical and Dental University, 1-5-45 Yushima, Bunkyo-ku, Tokyo 113-8510, Japan. ²Department of Biochemistry and Biophysics, Tokyo Medical and Dental University, Tokyo, Japan. ${ }^{3}$ Department of Joint Surgery and Sports medicine, Tokyo Medical and Dental University, Tokyo, Japan. ${ }^{4}$ Department of Cartilage Regeneration, Tokyo Medical and Dental University, Tokyo, Japan. ${ }^{5}$ National Hospital Organization, Disaster Medical Center, Tokyo, Japan. 
Received: 5 November 2017 Revised: 4 April 2018

Accepted: 12 April 2018 Published online: 02 May 2018

\section{References}

1. De Bari C, Dell'Accio F. Mesenchymal stem cells in rheumatology: a regenerative approach to joint repair. Clin Sci. 2007;113(8):339-48. https:// doi.org/10.1042/cs20070126.

2. Ullah I, Subbarao Raghavendra B, Rho GJ. Human mesenchymal stem cells current trends and future prospective. Biosci Rep. 2015;35(2):1-18. https:/ doi.org/10.1042/bsr20150025

3. De Bari C, Dell'Accio F, Tylzanowski P, Luyten FP. Multipotent mesenchymal stem cells from adult human synovial membrane. Arthritis Rheum. 2001; 44(8):1928-42. https://doi.org/10.1002/1529-0131(200108)44:8<1928::AIDART331>3.0.CO;2-P.

4. Wang $\mathrm{H}-\mathrm{S}$, Hung $\mathrm{S}-\mathrm{C}$, Peng $\mathrm{S}-\mathrm{T}$, Huang $\mathrm{C}-\mathrm{C}$, Wei H-M, Guo Y-J, et al. Mesenchymal Stem Cells in the Wharton's Jelly of the Human Umbilical Cord. Stem Cells. 2004;22(7):1330-7. https://doi.org/10.1634/stemcells.20040013.

5. Zuk PA. Human Adipose Tissue Is a Source of Multipotent Stem Cells. Mol Biol Cell. 2002;13(12):4279-95. https://doi.org/10.1091/mbc.E02-02-0105.

6. Dominici M, Le Blanc K, Mueller I, Slaper-Cortenbach I, Marini FC, Krause DS, et al. Minimal criteria for defining multipotent mesenchymal stromal cells. The International Society for Cellular Therapy position statement. Cytotherapy. 2006;8(4):315-7. https://doi.org/10.1080/14653240600855905.

7. Pittenger MF. Multilineage Potential of Adult Human Mesenchymal Stem Cells. Science. 1999;284(5411):143-7. https://doi.org/10.1126/science.284. 5411.143.

8. Calvi LM, Adams GB, Weibrecht KW, Weber JM, Olson DP, Knight MC, et al. Osteoblastic cells regulate the haematopoietic stem cell niche. Nature. 2003; 425(6960):841-6. https://doi.org/10.1038/nature02040.

9. Mitsiadis TA, Barrandon O, Rochat A, Barrandon Y, De Bari C. Stem cell niches in mammals. Exp Cell Res. 2007;313(16):3377-85. https://doi.org/10. 1016/j.yexcr.2007.07.027.

10. Crisan M, Yap S, Casteilla L, Chen C-W, Corselli M, Park TS, et al. A Perivascular Origin for Mesenchymal Stem Cells in Multiple Human Organs. Cell Stem Cell. 2008;3(3):301-13. https://doi.org/10.1016/j.stem.2008.07.003.

11. Sakaguchi Y, Sekiya I, Yagishita K, Muneta T. Comparison of human stem cells derived from various mesenchymal tissues: Superiority of synovium as a cell source. Arthritis Rheum. 2005;52(8):2521-9. https://doi.org/10.1002/art. 21212.

12. Nakamura T, Sekiya I, Muneta T, Hatsushika D, Horie M, Tsuji K, et al. Arthroscopic, histological and MRI analyses of cartilage repair after a minimally invasive method of transplantation of allogeneic synovial mesenchymal stromal cells into cartilage defects in pigs. Cytotherapy. 2012; 14(3):327-38. https://doi.org/10.3109/14653249.2011.638912.

13. Hatsushika D, Muneta T, Nakamura T, Horie M, Koga H, Nakagawa Y, et al. Repetitive allogeneic intraarticular injections of synovial mesenchymal stem cells promote meniscus regeneration in a porcine massive meniscus defect model. Osteoarthr Cartil. 2014;22(7):941-50. https://doi.org/10.1016/j.joca. 2014.04.028

14. Sekiya I, Muneta T, Horie M, Koga H. Arthroscopic Transplantation of Synovial Stem Cells Improves Clinical Outcomes in Knees With Cartilage Defects. Clin Orthop Relat Res. 2015;473(7):2316-26. https://doi.org/10.1007/ s11999-015-4324-8.

15. Mizuno M, Katano H, Otabe K, Komori K, Matsumoto Y, Fujii S, et al. Plateletderived growth factor (PDGF)-AA/AB in human serum are potential indicators of the proliferative capacity of human synovial mesenchymal stem cells. Stem Cell Res Ther. 2015;6(1):243. https://doi.org/10.1186/s13287015-0239-2.

16. Nimura A, Muneta T, Koga H, Mochizuki T, Suzuki K, Makino H, et al. Increased proliferation of human synovial mesenchymal stem cells with autologous human serum: Comparisons with bone marrow mesenchymal stem cells and with fetal bovine serum. Arthritis Rheum. 2008:58(2):501-10. https://doi.org/10.1002/art.23219.

17. Smith MD. The Normal Synovium. Open Rheumatol J. 2011:5(1):100-6. https://doi.org/10.2174/1874312901105010100

18. Nakagawa Y, Muneta T, Otabe K, Ozeki N, Mizuno M, Udo M, et al. Cartilage Derived from Bone Marrow Mesenchymal Stem Cells Expresses Lubricin In Vitro and In Vivo. PLoS One. 2016;11(2):e0148777. https://doi.org/10.1371/ journal.pone.0148777.
19. Schneider CA, Rasband WS, Eliceiri KW. NIH Image to ImageJ: 25 years of image analysis. Nat Methods. 2012;9(7):671-5. https://doi.org/10.1038/ nmeth.2089

20. Kohno Y, Mizuno M, Ozeki N, Katano H, Komori K, Fujii S, et al. Yields and chondrogenic potential of primary synovial mesenchymal stem cells are comparable between rheumatoid arthritis and osteoarthritis patients. Stem Cell Res Ther. 2017;8 https://doi.org/10.1186/s13287-017-0572-8.

21. Wang Q, Rozelle AL, Lepus CM, Scanzello CR, Song JJ, Larsen DM, et al. Identification of a central role for complement in osteoarthritis. Nat Med. 2011;17(12):1674-9. https://doi.org/10.1038/nm.2543.

22. Roelofs AJ, Zupan J, Riemen AHK, Kania K, Ansboro S, White N, et al. Joint morphogenetic cells in the adult mammalian synovium. Nat Commun. 2017:8:15040. https://doi.org/10.1038/ncomms15040.

23. Mabuchi Y, Morikawa S, Harada S, Niibe K, Suzuki S, Renault-Mihara F, et al. LNGFR+THY-1+VCAM-1 hi+ Cells Reveal Functionally Distinct Subpopulations in Mesenchymal Stem Cells. Stem Cell Rep. 2013;1(2):152-65. https://doi.org/ 10.1016/j.stemcr.2013.06.001

24. Churchman SM, Ponchel F, Boxall SA, Cuthbert R, Kouroupis D, Roshdy T, et al. Transcriptional profile of native CD271+ multipotential stromal cells: Evidence for multiple fates, with prominent osteogenic and Wnt pathway signaling activity. Arthritis Rheum. 2012;64(8):2632-43. https://doi.org/10. 1002/art.34434.

25. Ogata Y, Mabuchi Y, Yoshida M, Suto EG, Suzuki N, Muneta T, et al. Purified Human Synovium Mesenchymal Stem Cells as a Good Resource for Cartilage Regeneration. PLoS One. 2015;10(6):e0129096. https://doi.org/10. 1371/journal.pone.0129096.

26. Quirici N, Soligo D, Bossolasco P, Servida F, Lumini C, Deliliers GL. Isolation of bone marrow mesenchymal stem cells by anti-nerve growth factor receptor antibodies. Exp Hematol. 2002;30(7):783-91. https://doi.org/10. 1016/s0301-472x(02)00812-3.

27. Morito T, Muneta T, Hara K, Ju YJ, Mochizuki T, Makino H, et al. Synovial fluid-derived mesenchymal stem cells increase after intra-articular ligament injury in humans. Rheumatology. 2008:47(8):1137-43. https://doi.org/10. 1093/rheumatology/ken114.

28. Matsukura Y, Muneta T, Tsuji K, Koga H, Sekiya I. Mesenchymal stem cells in synovial fluid increase after meniscus injury. Clin Orthop Relat Res. 2014; 472(5):1357-64. https://doi.org/10.1007/s11999-013-3418-4.

29. Moseti D, Regassa A, Kim W-K. Molecular Regulation of Adipogenesis and Potential Anti-Adipogenic Bioactive Molecules. Int J Mol Sci. 2016;17(1) https://doi.org/10.3390/ijms17010124.

30. Caplan Al. All MSCs Are Pericytes? Cell Stem Cell. 2008;3(3):229-30. https:// doi.org/10.1016/j.stem.2008.08.008.

\section{Ready to submit your research? Choose BMC and benefit from:}

- fast, convenient online submission

- thorough peer review by experienced researchers in your field

- rapid publication on acceptance

- support for research data, including large and complex data types

- gold Open Access which fosters wider collaboration and increased citations

- maximum visibility for your research: over $100 \mathrm{M}$ website views per year

At BMC, research is always in progress.

Learn more biomedcentral.com/submissions 